Nouvelles perspectives en sciences sociales

\title{
La mondialisation : vers une compréhension duelle de l'homogénéisation et de la différenciation
}

\section{Roger Gervais}

Volume 2, numéro 1, septembre 2006

URI : https://id.erudit.org/iderudit/602455ar

DOI : https://doi.org/10.7202/602455ar

Aller au sommaire du numéro

\section{Éditeur(s)}

Prise de parole

\section{ISSN}

1712-8307 (imprimé)

1918-7475 (numérique)

Découvrir la revue

\section{Citer cet article}

Gervais, R. (2006). La mondialisation : vers une compréhension duelle de l'homogénéisation et de la différenciation. Nouvelles perspectives en sciences sociales, 2(1), 69-100. https://doi.org/10.7202/602455ar 


\title{
La mondialisation : vers une compréhension duelle de l'homogénéisation et de la différentiation
}

\author{
Roger Gervais \\ Université de Toulouse 1 \\ CIRESS - LEREPS - GRES, France
}

Selon plusieurs observateurs, la culture états-unienne s'impose à celle de bon nombre de pays, surtout depuis l'avènement d'Internet et de l'accélération de la tendance à la mondialisation qui en est le corollaire. Mais cette observation est-elle juste ? Peut-on craindre que l'avenir prenne la forme d'une espèce de melting pot culturel mondial ? Pour bon nombre de peuples, il s'agit là d'une menace réelle, menace qui ne dissipe en rien la campagne américaine de dite démocratisation des pays arabes.

Selon certains auteurs qui font état de comportements de masse ou encore de l'influence des médias, souvent en s'inspirant de l'esprit des travaux d'Adorno et Horkheimer ${ }^{1}$, cette homogénéisation culturelle est

1 Théodor Adorno et Max Horkheimer, La dialestique de la raison : fragments philosophiques, Paris, Gallimard, 1974, p. 281. 
théoriquement plausible ${ }^{2}$. Selon d'autres, qui observent par exemple la formation de contre-cultures ou l'accentuation de l'individualisme, il est préférable de parler de régionalisation ${ }^{3}$, de différenciation ou même d'éclatement des cultures ${ }^{4}$. Les réflexions qui s'alimentent parfois à la systémique complexe, avancent plutôt que ces deux pôles devraient être compris comme les manifestations d'un mouvement duel, la première de ces manifestations étant responsable de la reproduction de la société et, la seconde, de son évolution. Cette dualité ne donnerait lieu à la négation d'aucune de ses composantes, malgré l'influence de la mondialisation ${ }^{5}$. L'interprétation du devenir des sociétés postindustrielles peut ainsi prendre plusieurs voies.

\section{Hypothèses}

L'objectif premier de notre travail était de vérifier l'hypothèse d'une contradiction entre l'homogénéisation et l'hétérogénéisation sur le plan macrosocial en faisant reposer l'analyse sur des données empiriques. Il faut cependant noter que nous ne voulions fonder cette recherche ni sur un modèle déterministe, ni sur une approche psychologisante ; les impasses de ces modèles sont bien connues et on sait désormais leur

2 Voir, par exemple, Jean Baudrillard, Amérique, Paris, Grasset et Fasquelle, 1986, p. 249; Claude Karnoouh, Adieu à la différence : essais sur la modernité tardive, Paris, Arcantère Karnoouh, 1993, p. 228 ; Manuel Castells, La société en réseaux : l'ère de linformation, Paris, Fayard, 1998, p. 613 ; Gary Teeple, Globalization and the Decline of the Social Reform : Into the Twenty-first Century, Aurora, Garamond Press, 2000, p. 239.

3 Voir, par exemple, Philippe Breton, « La société de l'information : de l'utopie au désenchantement », Revue européenne des sciences sociales, Tome XL, n 123, 2002, p. 3539 ; Isabelle Paillart, «La société de l'information : une société de contradictions ? 》, Revue européenne des sciences sociales, Tome XI, $n^{\circ} 123,2002$, p. 55-63.

4 Voir, entre autres, Alain Touraine, Pourrons-nous vivre ensemble? Égaux et différents, Paris, Librairie Arthème Fayard, 1997, p. 395 ; Yves, Boisvert, «Sortir du nihilisme : Quand la dictature du moi sert de bouée », dans Yves Boisvert et Lawrence Olivier (dir.), $\grave{A}$ chacun sa quête : essais sur les nouveaux visages de la transcendance, Québec, Presses de l'Université du Québec, 2000, p. 1-10; Bernard Gagnon, « Le soi et le différent à l'âge de l'indifférence : la problématique de l'éducation ", dans Yves Boisvert et Lawrence Olivier (dir), Á chacun sa quête : essais sur les nouveaux visages de la transcendance $Q u e ́ b e c$, Presses de l'Université de Québec, 2000, p. 86-103.

5 Voir, par exemple, Edgar Morin, Introduction à la pensée complexe, Paris, ESF éditeur, 1990, p. 158 ; Simon Laflamme et Ali Reguigui, Homogénéité et distinction, Sudbury, Prise de parole, 2004, p. 255. 


\section{ROGER GERVAIS / LAMONDIALISATION}

incapacité à accéder à la complexité du social. Nous proposions plutôt de recourir au modèle de la circulation des biens, des idées et des personnes, tel qu'il a été développé par Laflamme ${ }^{6}$. Ce modèle permet de sortir des impasses et présente les composantes grâce auxquelles on peut mesurer le degré d'homogénéisation et le degré d'hétérogénéisation qui sont liés à la mondialisation.

Étant donné que ce modèle comporte des éléments de la sociologie, de l'économie et des sciences de la communication, il est fort difficile d'en rapporter, dans le détail, toutes ses composantes théoriques. Il nous semble néanmoins utile de tenter une synthèse en quatre points :

1) Le modèle s'insère dans une approche constructiviste, c'est-à-dire que l'auteur est conscient que les réalités sociales - par exemple, les individus, les institutions, les discours sociaux, etc. - sont tellement interreliées que seule l'abstraction en permet une conceptualisation catégorielle. Le modèle ne tente pas d'expliquer le social dans sa totalité ${ }^{7}$ ni d'imposer de vérité absolue. Il admet que les catégories et leurs relations produisent leur objet dialectiquement à travers un moment précis de l'histoire.

2) Le modèle prend appui sur les principes d'échange ${ }^{8}$, de communication et de relation; ce sont eux qui donnent leur sens à une catégorisation en termes de biens, d'idées et de personnes. « [C]es trois notions [biens, idées et personnes] ont pour connotation des rapports que peuvent entretenir entre eux des agents : les sociétés, est-il affirmé par là, se reproduisent et se produisent par les interactions qui les animent ${ }^{9}$. De plus, le modèle considère que ces notions doivent quitter la forme antesociale puisqu'elles se produisent au sein de dynamiques relationnelles.

6 Simon Laflamme, La société intégrée : de la circulation des biens, des idées et des personnes, New York, Peter Lang, 1992, p. 310 ; Simon Laflamme, Des biens, des idées et des personnes au Canada (1981-1995) : un modele macrologique relationnel, Paris/Sudbury, L'Harmattan/ Prise de parole, 2000, p. 273.

7 Simon Laflamme, La société intégrée..., op. cit, p. 256.

8 «De Adam Smith à Friedman, de Wiener à Habermas ou à Élias, de Mauss à Caillé, les phénomènes économiques, communicationnels ou sociologiques se soumettent à des interprétations où est mis en ouvre ce principe d'échange " (Simon Laflamme, Des biens, des idées et des personnes au Canada (1981-1995)..., op. cit., p. 12).

'Simon Laflamme, La société intégrée..., op. cit., p. 3-4. 
3) Comme conséquence de cette logique relationnelle, le modèle a recours à la notion de dialectique, même de trialectique - puisqu'il y a une relation entre trois catégories. Essentiellement, c'est la dynamique relationnelle qui domine le modèle et non une perspective unilatérale, de cause à effet.

4) Le modèle s'estime capable d'observer la société dans une logique complexe, d'accéder à la concentration (échange restreint) et à la déconcentration (échange étendu) qui caractérisent les liens entre les trois lieux de la circulation que sont ceux des biens, des idées et des personnes. Amélioré pour nos fins, cela signifie que si l'échange d'une catégorie en relation avec les autres catégories est étendu, il y aura homogénéisation et le modèle sera en mesure de tenir compte de son importance. Si, au contraire, l'échange d'une catégorie en relation avec les deux autres est restreint, il y aura hétérogénéisation, et le modèle sera en mesure de tenir compte de son importance. S'il y a échange étendu et restreint d'une catégorie en relation avec les autres, il y aura et homogénéisation et hétérogénéisation à la fois. Le modèle sera alors en mesure de repérer cette situation contradictoire.

Le modèle se veut donc constructiviste en tant que l'empirie est produite par les catégories - elles-mêmes construites et en construction - qui sont manipulées ; en ce sens, il ne peut pas prétendre à l'exhaustivité. Il se fonde sur l'échange, sur la communication et il entend ainsi privilégier les dynamiques entre les objets plutôt que les objets en eux-mêmes. Il est trialectique puisqu'il ne veut pas qu'une catégorie ait une plus grande importance que les deux autres et parce qu'il fait en sorte que chaque catégorie soit toujours comprise dans la dialectique qu'elle entretient avec les deux autres catégories elles-mêmes dialectisées. Cela signifie que les personnes font circuler des idées en relation avec des biens, que des biens font circuler des idées en relation avec des personnes et que des idées font circuler des personnes en relation avec des biens ${ }^{10}$. Ainsi, le modèle arrive à comprendre le degré

10 Prenons les diverses idées liées aux régimes amaigrissants. Même si ce sont des personnes qui ont conçu les régimes et qui les ont diffusés au grand public, ces idées sont présentement en circulation. La télévision en parle. Les journaux en parlent. Les individus en parlent en prenant un café, ou dans l'autobus, et nous les entendons, c'est-à-dire que nous entendons les idées qui circulent. Certains entendent parler de Weight Watchers, d'autres de Jenny Craig, d'autres de Slim Fast, ou d'Atkins, ou de South Beach, etc. Certains suivent tel régime fidèlement ou tel autre. Certains en 
de cette circulation, donc de l'étendue de l'homogénéisation et de l'hétérogénéisation.

Ces assises théoriques viennent combler les lacunes des modèles unilatéraux et anthropocentriques et permettent de comprendre la société en respectant davantage sa complexité. De plus, étant donné que notre recherche a pour arrière-fond la mondialisation, le modèle est bénéfique par le fait qu'il intègre des catégories essentiellement rattachées à ce phénomène : les biens, les idées et les personnes. L'utilisation d'au moins deux catégories en même temps, soit celles des idées et des personnes ${ }^{11}$, abordées dans une approche relationnelle, et l'utilisation de certains concepts comme celui de concentration (une circulation restreinte des biens, des idées ou des personnes) et de déconcentration (une circulation ouverte des biens, des idées et des personnes) font en sorte que le modèle que nous empruntons et aménageons devrait permettre de jeter quelque lumière sur notre problématique et de répondre, plus particulièrement, à la question suivante : la mondialisation conduit-elle vers l'homogénéisation des

suivent un et changent pour un autre. Certains ne suivent aucun régime. Ce qu'il importe de retenir, c'est que ce ne sont pas nécessairement les personnes qui s'échangent les idées liées aux régimes, mais que ce sont les idées liées aux régimes qui s'échangent des personnes. Par exemple, le régime Weight Watchers (idée) échange des personnes avec le régime d'Atkins (une autre idée) ou le régime de Jenny Craig échange des personnes avec Slim Fast, et ainsi de suite. Nous pourrions même dire que certains régimes s'échangent des personnes avec des non-régimes. Même si notre conception anthropocentrique voudrait nous faire dire que les personnes changent de régimes, donc changent d'idée et que cela fait en sorte qu'il y a des personnes qui échangent des idées, cette affirmation serait aussi relative que celle qui veut que les idées échangent des personnes qui sont en relation. Cela reviendrait à dire, au fond, que les personnes s'échangent des idées, aussi bien que les idées s'échangent des personnes. C'est donc une question de " point de vue " et la compréhension de cette relativité permet de mieux cerner la complexité du social : un phénomène qui n'a pas simplement pour centre les personnes. Nous pouvons aussi faire cette même opération rationnelle avec les biens en relation avec les personnes ou les idées; le modèle de la circulation des biens, des idées et des personnes le fait avec ces trois composantes dans leur relation trialectique afin de saisir le plus d'informations possible au sujet d'une société, dans le contexte des catégories qu'il manipule.

11 Il aurait été très utile d'intégrer dans cette recherche les trois catégories comme le veut le modèle de Laflamme. Toutefois, une telle opérationnalisation aurait été trop exigeante dans le cadre de cette étude, d'autant plus que nos hypothèses pouvaient être vérifiées sans que nous satisfassions à une telle exigence. 
individus, des cultures et des groupes sociaux ou, à l'inverse, oriente-telle vers l'hétérogénéisation?

Les hypothèses peuvent donc être formulées comme suit.

1. Si la thèse de l'homogénéisation est vraie, plus nous avançons dans le temps, plus nous devrions observer de déconcentration entre les idées et les personnes, peu importe la ville à l'étude.

2. Si la thèse de l'hétérogénéisation est vraie, plus nous avançons dans le temps, plus nous devrions observer de concentration entre les idées et les personnes, quelle que soit la ville que nous étudions.

3. Si la mondialisation est homogénéisation, plus nous avançons dans le temps, plus nous devrions observer de similitudes entre les villes.

4. Si la mondialisation est hétérogénéisation, plus nous avançons dans le temps, plus nous devrions observer de différences entre les villes.

\section{2. Échantillonnage}

Afin d'opérationnaliser le modèle de la société intégrée, Laflamme (2000) avait choisi d'analyser la circulation des biens, des idées et des personnes au Canada entre 1981 et 1995. Il exploitait ainsi les catégories du modèle au sein d'un même pays et l'analyse se faisait sur une période de quinze ans. Le fait de l'homogénéisation, tel qu'associée à la mondialisation, force à étendre l'analyse. Pailliart reproche au discours sur l'homogénéisation de ne tenir compte ni de l'hétérogénéité régionale, ni de la dimension infranationale ${ }^{12}$. Il nous semble essentiel d'aborder le phénomène selon ces deux dimensions. Miège critique la validité de la thèse de Castells, une thèse qui voudrait que les sociétés soient réseautées depuis l'avènement d'Internet. Selon Miège, il est impossible de cerner les effets de l'informatisation sans tenir compte des années précédant 1980 ; ce que Castells dit faire, mais ce que, en réalité, il ne fait pas $^{13}$. Cette critique nous concerne directement puisque Internet est considéré comme l'un des facteurs qui ont favorisé la

12 Isabelle Paillart, la Société de linformation..., op. cit.

13 Bernard Miège, «La Société de l'information : toujours aussi inconcevable », Revue européenne des sciences sociales, Tome XL, $\mathrm{n}^{\circ} 123,2002$, p. 47. 
mondialisation des idées. Nous nous voyons, de ce fait, dans l'obligation d'étendre la période analysée afin de tenir compte de la circulation des biens, des idées et des personnes avant l'avènement d'Internet, c'est-àdire en 1960, et de comparer l'intégration des biens, des idées et des personnes de cette époque avec une intégration plus contemporaine pour vérifier si elle est de même nature.

Il n'est évidemment pas possible d'observer le phénomène qui nous intéresse de façon universelle aux plans international et infranational. Il est même compliqué de le faire dans un registre limité en manipulant les trois catégories les plus empiriques du modèle. Nous proposons d'entreprendre le projet en comparant la circulation des idées et des personnes dans deux villes, situées toutes les deux dans un pays différent. Pour ce faire, nous avons arrêté notre choix sur la Ville du Grand Sudbury, dans le nord de l'Ontario, au Canada, et sur Toulouse, dans la région administrative du Midi-Pyrénées, dans le sud-ouest de la France. Nous avons aussi défini deux périodes différentes, soit les mois d'août, septembre et octobre, en 1960 et en 2000. La collecte de données a porté sur un quotidien et un hebdomadaire pour chacune des deux villes.

\subsection{Choix des deux villes}

Nous avons choisi ces deux villes parce qu'elles sont, toutes les deux, des centres régionaux importants (Sudbury pour le nord de l'Ontario; Toulouse pour le sud-ouest de la France). De plus, chacune d'elles contribue considérablement au secteur économique de sa région (Sudbury avec ses mines de nickel, Toulouse avec ses industries aéronautiques et spatiales).

Certains nous reprocheront d'avoir introduit un biais dans nos résultats en choisissant deux villes très différentes. Par exemple, la Ville du Grand Sudbury a une population de 155220 habitants, alors que Toulouse en compte 398500 ; Sudbury est une ville majoritairement anglophone avec une forte minorité francophone (environ $30 \%$ de la population), tandis que Toulouse est presque exclusivement francophone. Et ce ne sont là que quelques différences parmi d'autres. Toutefois, nous aimerions rappeler que ces dissimilitudes sont précisément ce dont fait état Pailliart. Deux villes auront toujours des différences considérables - ce pour quoi il nous semble que même un choix de villes théoriquement semblables prêterait à la critique. Mais déclarer qu'il n'y aura que des différences, c'est négliger a priori la part de l'homogénéisation transnationale. C'est en comparant ces deux villes qu'il sera possible de vérifier l'hypothèse du 
" régionalisme » de Pailliart, certes, mais aussi celle pour laquelle l'évolution des sociétés contemporaines est à la fois homogénéisation et hétérogénéisation, aux plans tant international qu'infranational.

\subsection{Choix des années}

Nous avons choisi de nous arrêter sur l'ensemble des articles liés à des problématiques politiques parus pendant une période de trois mois en 1960 et, à nouveau, en 2000, et cela, pour des périodiques retenus pour chacune des régions. Des périodes de trois mois nous semblaient suffisantes pour observer des récurrences dans les problématiques politiques. Le choix des mois d'août, septembre et octobre s'explique par le fait que nous croyons que cette période de l'année est la plus mouvementée sur le plan politique - la fin de l'été appelle les questions liées au tourisme ; la rentrée scolaire suscite des questions liées à l'éducation ; le dernier trimestre fiscal de l'année appelle les questions liées à l'économie, etc. Nous avons choisi 1960 afin d'observer une époque où la société ne fait pas encore un usage massif d'Internet et 2000 puisque Internet y est fort utilisé. Un tel échantillon nous a paru d'autant plus important qu'il tient compte de l'aspect infranational, comme l'exige Palliart, et qu'il se donne un écart de temps assez important pour éviter les critiques de Miège.

\subsection{Choix des journaux}

Laflamme repère les « idées » dans les périodiques par référence à la notion de problématique politique ${ }^{14}$. Il retient ces médias pour deux raisons principales :

1) En étant en " relation d'interdépendance " avec les acteurs - les médias puisant leurs informations chez les acteurs et les acteurs s'informant à partir de ce qui est diffusé par les médias ${ }^{15}$-, les médias permettent de cerner les problématiques qui préoccupent les acteurs;

2) De par leur format écrit, les périodiques sont plus faciles à analyser que les autres formes de médias, telle la télévision.

14 Une problématique politique renvoie aux sujets ou aux discours sociaux qui, entre autres, reviennent fréquemment.

15 Cette perception des médias se trouve aussi chez Niklas Luhmann, The Reality of the Mass Media, California, Standford University Press, 2000, p. 154. 
Dans chaque région, nous avons retenu un quotidien et un hebdomadaire qui étaient présents en 1960 et 2000. Le fait d'avoir au moins deux périodiques permet d'assurer une meilleure représentativité des idées qui circulent à un moment donné dans le lieu étudié. Si les deux périodiques permettent le repérage d'idées semblables, nous pourrons affirmer que ces données témoignent relativement bien des problématiques politiques de la région, et moins des intérêts spécifiques d'un éditeur, par exemple.

Dans chacun des quotidiens, ont été retenus pour l'analyse, quatre jours qui sont les plus proches de la parution de l'hebdomadaire à toutes les quatre semaines entre les mois d'août et d'octobre - par exemple, quatre jours durant la semaine du 4 au 16 septembre et, ensuite, quatre jours pendant la semaine du 2 au 8 octobre. Cela permettrait d'établir le contexte dans lequel se présentaient les idées dégagées à partir de l'hebdomadaire. Nous avons examiné l'hebdomadaire toutes les semaines afin de suivre le fil des idées diffusées dans le quotidien - en d'autres mots, afin de veiller à ce que les idées qui se dégagent du quotidien ne soient pas strictement événementielles. Les informations provenant de chacun des journaux se complètent les unes les autres: le quotidien permet d'établir le contexte dans lequel s'inscrit l'hebdomadaire, et celui-ci établit les liens de continuité entre les idées qui proviennent du quotidien.

Le quotidien de Sudbury est The Sudbury Star (1960 et 2000); à Toulouse, il s'agit de La Dépêche du Midi (1960 et 2000). Comme hebdomadaire, à Sudbury, nous avons choisi l'Ami du peuple (1960) qui devient Le Voyageur (2000); à Toulouse, nous avons retenu La Croix de Toulouse (1960), qui devient La Croix du Midi (2000). Le quotidien et l'hebdomadaire de Sudbury ne sont pas de même langue - le premier est en anglais, le second, en français ; ceux de Toulouse sont de langue française uniquement. Nous avons jugé important d'inclure le journal francophone de Sudbury dans notre échantillon afin de représenter la forte minorité de francophones à laquelle ce journal est destiné. À Toulouse, les journaux francophones sont presque exclusifs.

\section{Méthode de collecte}

Dans De la Circulation des biens, des idées et des personnes au Canada (19811995) : un modèle macrologique relationnel (2000), Laflamme procède à une espèce d'herméneutique. Toutefois, pour ce genre de travail, nous 
croyons qu'un traitement quantitatif est plus prudent de par le fait qu'il diminue la possibilité de fausses représentations. Pour ne pas prêter le flanc à certaines critiques que pourrait soulever cette orientation, nous intégrons aussi certains éléments de l'analyse qualitative. Par exemple, la grille d'analyse n'impose pas les thèmes (idées) ${ }^{16}$ à repérer lors du recensement des articles ; c'est la récurrence qui constitue le principal indicateur d'un thème à la première étape du travail. De plus, chaque thème est présenté dans le contexte qui a permis sa construction. Nous croyons que ces procédures méthodologiques minimisent les motifs des critiques d'un travail purement quantitatif en maintenant, le plus possible, la complexité des résultats.

\subsection{Construire des fiches d'analyse}

À partir des articles retenus, nous avons repéré les informations suivantes : la date de parution du journal, le titre du journal, le titre de l'article, l'auteur de l'article, le genre d'article (reportage, éditorial, lettre à l'éditeur, lettre d'opinion, chronique culturelle ou chronique santé, etc.), la catégorie géopolitique des événements (les événements dont fait état l'article se sont déroulés au plan international, national, provincial ou régional) ; nous avons aussi résumé chacun des articles. Le tout a été inséré dans des fiches de Merge files en Corel WordPerfect afin de faciliter la gestion des données.

\subsection{Identifier les thèmes ou les idées}

Certains articles ont plus d'une thématique et parfois les thèmes ne sont pas toujours mutuellement exclusifs - il arrive qu'un journal présente des idées regroupées à un moment et dissociées à d'autres. Le dénombrement

16 Dans le travail original (Roger Gervais, Les effets de la mondialisation sur la relation entre les idées et les personnes : une comparaison entre les régions toulousaine et sudburoise, mémoire de maîtrise, Sudbury, Université Laurentienne, 2005, p. 132), nous avions constaté que l'utilisation du mot « idée » portait parfois à confusion. À certains moments, le terme se référait au concept catégorique du modèle de Laflamme. À d'autres moments, il se référait à une opinion dégagée en tant qu'unité informationnelle. À d'autres encore, il se référait à une catégorisation de plusieurs opinions semblables. Nous avons tenté de diminuer cette confusion en utilisant le mot « thème » pour les idées catégorisées. Cette manière de faire semble diminuer l'ambiguïté entre le concept de Laflamme et le mot compris dans son sens plus usuel. 
des thèmes s'effectue donc indépendamment de celui des autres. Si, en parlant des Nazis, les journaux toulousains les associent parfois aux Allemands, il devient nécessaire de compter l'idée dans la catégorie « nazis » et aussi dans la catégorie «Allemands ». Si, au contraire, les journaux présentent les Nazis comme un groupe distinct, sans mentionner les Allemands, on ne compte alors l'idée que sous la catégorie « nazis ». Ce genre de scénario a tendance à se produire au sein d'articles qui abordent des sujets rattachés à des leaders politiques : parfois, le rôle de représentant d'un pays ou l'adhésion à une position politique est visiblement discernable et nécessite un premier dénombrement pour le leader et un autre pour le pays ou pour la position politique; parfois, le leader est présenté comme un individu puissant et capable de penser hors du cadre de son pays ou d'une position politique. Ainsi, en tout temps, c'est le contexte qui détermine l'inscription des idées à l'intérieur d'un thème.

\subsection{Identifier les tons associés aux thèmes}

En correspondance avec l'étape précédente, les tons associés aux thèmes ont été repérés selon deux possibilités : un ton positif renvoie à la valorisation du thème, un ton négatif, à sa critique. Il ne faut pas confondre le ton positif ou négatif et le simple fait de dire oui ou non ou d'indiquer qu'une chose est bonne ou mauvaise, puisque les tons ont été définis à partir du contexte dans lequel s'inscrivent les idées. Conformément à cet esprit, un thème présenté avec un ton neutre, respectant la neutralité journalistique, a été défini selon la connotation sociale qui se dégage des autres textes. Par exemple, malgré la neutralité journalistique, un article sur la criminalité peut avoir un ton négatif s'il est entouré d'articles qui témoignent d'une aversion pour l'acte criminel ; la pauvreté, présentée de façon prétendument neutre, peut donner cours à un ton négatif s'il y a suffisamment d'articles qui témoignent d'une aversion pour l'acte criminel ; la pauvreté, présentée de façon prétendument neutre, peut donner cours à un ton négatif puisqu'il y a suffisamment de textes qui témoignent d'un effort collectif pour atténuer ce phénomène au sein des sociétés; les contributions sociales, présentées avec une neutralité journalistique, peuvent révéler un ton positif dans le cadre où il y a toujours des articles qui louent les 
gestes de bienfaisance et jamais d'articles qui ne les dénoncent ${ }^{17}$. La méthode utilisée pour définir les idées neutres à partir de la symbolique sociale doit toujours renvoyer au contexte que présente le journal ; ce sont les autres articles qui permettront de savoir si une idée neutre a une connotation positive ou négative. Le ton ne peut donc pas être défini sans qu'il soit justifié par le contexte qui se dégage du journal. Toute idée doit être présentée à plusieurs reprises avant qu'elle ne soit classifiée et elle l'est toujours en vertu de cette récurrence. Ainsi, la classification doit être justifiée, récurrence et contexte inclus, afin qu'on puisse saisir la logique qui l'a autorisée. Cette justification, toujours explicite, prendra cette forme :

AFRIQUE :

Quatre-vingt-quinze pour cent des articles qui, dans le The Sudbury Star, touchent aux questions liées au continent africain signalent l'état de besoin dans lequel se trouvent les divers pays qui le constituent. La situation économique défavorable de ces pays, l'état de santé des populations, le manque de denrées alimentaires, la guerre et le besoin de soutien composent l'image que dessine le journal, soit 40 articles dont le ton est négatif. Les deux articles au ton positif (5\%) félicitent les gouvernements africains qui cherchent à mener les pays vers une autonomie, une indépendance à l'égard des pays coloniaux, à l'image du Canada. De plus, ils mentionnent le fait qu'en atteignant l'indépendance, ces pays pourront devenir des alliés des pays occidentaux et renforcer leurs positions au sein de l'ONU afin de faire face à la montée du communisme.

L'Ami du peuple consacre 11 articles à ce même sujet, 9 d'un ton négatif, 2 d'un ton positif. Les raisons évoquées dans The Sudbury Star sont les mêmes que celles de l'Ami du peuple.

17 Certains auteurs en analyse du discours, notamment Maingueneau, suggèrent plusieurs opérations méthodologiques efficaces pour ce genre de travail. La taille de notre échantillon et l'ampleur des informations repérées, par contre, rendent difficile la mise en pratique de ces suggestions méthodologiques qui ont, malgré tout, inspiré ce travail. Voir Dominique Maingueneau, Genèses du discours, Liège, Mardaga, 1984, p. 209. 
Nous avons choisi de discriminer selon le ton - positif ou négatif puisque nous croyons que cet indice, nonobstant les critiques qu'on peut en faire, est facilement exploitable et donne lieu à une classification claire et simple.

\subsection{Dégager le degré de concentration pour chaque section} échantillonnale - comprendre la circulation des idées selon les personnes au sein d'un journal

Les idées, sans considération de leur ton, ont été insérées dans une matrice de Statistical Program for Social Sciences (SPSS). Ce logiciel nous a permis de découvrir celles qui occupaient au moins $1 \%$ du total des idées dégagées dans un journal en particulier ${ }^{18}$. Ces idées identifiées, le ton leur a été associé de nouveau et elles ont été insérées dans un tableau tel le suivant.

\section{Tableau 1}

Nombre de thèmes selon le journal, le ton et l'année de parution

\begin{tabular}{|l|c|c|c|c|}
\hline \multirow{2}{*}{ Idées } & \multicolumn{2}{|c|}{ Journal X de Ville X 1960 } & \multicolumn{2}{c|}{ Journal Y de Ville X 1960 } \\
\cline { 2 - 5 } & Ton positif & Ton négatif & Ton positif & Ton négatif \\
\hline IDÉE X & 17 & 1 & 39 & 29 \\
IDÉE Y & 3 & 23 & 2 & 1 \\
IDÉE Z & 5 & 18 & 8 & 23 \\
\hline \multicolumn{4}{|l}{ Fréquences fictives présentées à des fins d'illustration seulement } \\
\hline
\end{tabular}

Les idées, en tant que thèmes, ont été insérées dans la première colonne, le titre du quotidien dans la deuxième colonne et le titre de l'hebdomadaire dans la troisième. Ces deux dernières colonnes, soit la colonne 2 et la colonne 3 , ont été divisées en deux afin d'y insérer la somme des tons positifs et négatifs, respectivement, pour chaque idée repérée lors des étapes précédentes.

18 Ce nombre paraitt peut-être peu significatif ; toutefois, à titre d'exemple, au cours des trois mois de la recension de 1960, 1040 idées ont été dégagées à partir du Sudbury Star seulement. Or, une proportion de $1 \%$ fait en sorte qu'une idée, pour être retenue, doit se manifester au moins à dix reprises. 
Cette première présentation des données ne permet ni la comparaison des idées entre elles, ni la comparaison des journaux entre eux puisque le nombre d'articles peut varier d'un journal à l'autre et le nombre d'idées peut varier d'un article à l'autre. En procédant à une telle comparaison, de façon stricte, nous commettrions une erreur de principe.

Ainsi, afin de permettre ce genre de comparaison, il était nécessaire de standardiser les nombres bruts en les transformant en valeurs relatives (voir le tableau 2$)^{19}$.

\section{Tableau 2}

Pourcentages des thèmes selon le journal, le ton et l'année de parution

\begin{tabular}{|l|c|c|c|c|}
\hline \multirow{2}{*}{ Idées } & \multicolumn{2}{|c|}{ Journal X de Ville X 1960 } & \multicolumn{2}{c|}{ Journal Y de Ville X 1960 } \\
\cline { 2 - 5 } & Ton positif & Ton négatif & Ton positif & Ton négatif \\
\hline IDÉE X & 0,94 & 0,06 & 0,57 & 0,43 \\
IDÉE Y & 0,12 & 0,88 & 0,66 & 0,43 \\
IDÉE Z & 0,22 & 0,78 & 0,26 & 0,74 \\
\hline
\end{tabular}

Pourcentages fictifs présentés à des fins d'illustration seulement, mais en correspondance avec le tableau 1

Cette standardisation a ensuite permis l'utilisation d'une Échelle de concentration, comme celle ci-dessous ${ }^{20}$.

\begin{tabular}{|c|c|c|c|c|c|c|c|c|c|}
\hline \multicolumn{1}{|c|}{ Échelle de concentration } \\
\hline- & --+ & +- & ++- & \multicolumn{2}{|c|}{+} & ++- & +- & -++ & - \\
\hline $0-10$ & $10-20$ & $20-30$ & $30-40$ & $40-50$ & $50-60$ & $60-70$ & $70-80$ & $80-90$ & $90-100$ \\
\hline
\end{tabular}

En application générale, le symbole négatif ( - ) équivaut à une idée qui est très déconcentrée, qui est partagée par la grande majorité d'une

19 Vu que chaque journal a un nombre total particulier d'articles, la standardisation en pourcentage est requise pour des fins de comparaison.

20 Cette échelle utilise les symboles que propose Laflamme, mais elle leur ajoute des valeurs quantifiées (Simon I aflamme, I a société intégrée..., op. cit.; Simon Laflamme, Des biens, des idées et des personnes au Canada (1981-1995), op. (it.). 
population $^{21}$. Dans cette recherche, pour qu'une idée soit catégorisée comme déconcentrée, elle doit nécessairement être présentée avec le même ton au moins $90 \%$ des fois où elle apparait. Le symbole de déconcentration ( - ) se trouve aussi à l'autre extrémité de l'échelle, soit à $10 \%$ ou moins, puisqu'une idée au ton $\mathrm{X}$, récurrent à $10 \%$ ou moins, implique que le ton opposé soit récurrent à $90 \%$ ou plus, donc qu'il soit celui de la grande majorité d'une population. Certains noteront possiblement que la valeur de $10 \%$ correspond à une concentration vraisemblable pour une idée donnée et que notre protocole ne permet pas d'en tenir compte. Malgré la pertinence de cette critique, nous croyons offrir un point de départ intéressant pour un travail de niveau macrologique ; miser sur la circulation d'informations " minoritaires » pourrait faire place à une toute autre forme d'analyse.

Le symbole positif $(+)$ équivaut à une idée très concentrée, ce qui permet l'identification de groupes distincts et spécifiques. En d'autres termes, si de 40 à $50 \%$ de la population partage une position et que le reste de la population partage la position opposée, deux groupes distincts et spécifiques peuvent être identifiés et le symbole de concentration doit être utilisé. Certains affirmeront peut-être que les idées qui provoquent des prises de position dichotomiques sont rares. Nous le reconnaissons, mais nous précisons que ce modèle ne présuppose pas des observations. Il doit être en mesure de vérifier une telle affirmation.

Lorsqu'une idée n'est pas partagée par la quasi-totalité de la population, et qu'elle ne permet pas la distinction de groupes spécifiques, elle n'est ni concentrée, ni déconcentrée $\left(+_{-}^{-}\right)$; nous l'observons entre 20 et $30 \%$ ou entre 70 et $80 \%$. Il aurait été aussi logique de nommer ce symbole concentré et déconcentré. Ce qui est important, par contre, c'est de ne pas confondre ce symbole avec le symbole plus généralement associé à la neutralité puisqu'il y a des prises de position à l'égard de ces idées ; elles ne sont, tout simplement, ni partagées par la quasi-totalité de la population, ni sources de polémiques polarisantes.

21 Il est important de rappeler ici que les articles qui sont diffusés au sein des journaux sont théoriquement représentatifs des idées ou des problématiques politiques qui circulent dans la population. C'est dans cet esprit que nous parlons de population. 
Les symboles qui qualifient une idée plus ou moins déconcentrée (- - +), soit entre 10 et $20 \%$ ou entre 80 et $90 \%$ des cas, ou ceux qui la qualifient de plus ou moins concentrée $(++-)$, soit entre 30 et $40 \%$ ou 60 et $70 \%$, correspondent aux niveaux intermédiaires. Le premier symbole apparaît dans le contexte où les groupes qui sont en relation avec une idée sont difficiles à distinguer, sans qu'il y ait homogénéisation totale ; l'idée en relation avec les personnes sera plus ou moins déconcentrée $(--+)$. Le deuxième $(++-)$ se rapporte plutôt à la situation où les groupes qui sont en relation avec une idée sont faciles à distinguer, sans qu'il y ait deux groupes entièrement polarisés; l'idée en relation avec les personnes est donc plus ou moins concentrée $(++-)$. Un troisième tableau a tenu compte de ces éventualités (voir tableau 3).

Tableau 3

Niveau de concentration selon le journal et la ville en 1960

\begin{tabular}{|l|c|c|}
\hline Idées & Journal X de Ville X 1960 & Journal Y de Ville X 1960 \\
\hline IDÉE X & - & + \\
IDÉE Y & -+ & ++ \\
IDÉE Z & -+ & +- \\
\hline
\end{tabular}

Symboles fictifs présentés à des fins d'illustration seulement, mais en correspondance avec le tableau 2

En nous référant à une situation fictive, nous décrivons la procédure à suivre pour monter des tableaux comme le précédent. L'idée $\mathrm{X}$ est présentée par le Journal $\mathrm{X}$ de la Ville $\mathrm{X}$ avec un ton positif à dix-sept reprises et un ton négatif à une reprise (voir le tableau 1). En pourcentage, cela donne $94 \%$ et $6 \%$. En faisant référence à l'échelle de concentration, il est possible de déterminer que l'idée $\mathrm{X}$ est très déconcentrée dans le Journal $\mathrm{X}$ de la ville $\mathrm{X}$ : $94 \%$ se situant entre 90 et $100 \% ; 6 \%$ entre 0 et $10 \%$. Le symbole de déconcentration ( - ) convenant le mieux dans ce cas-ci, c'est donc lui qui est inscrit dans le tableau 3. Nous procédons ainsi chaque fois de la même manière.

L'objectif du tableau 3 est de faciliter la compréhension de la relation entre les idées et les personnes pour chaque journal. Cette compréhension n'est possible que par déduction puisque, malgré l'échelle ordinale que nous avons associée aux symboles de Laflamme, ces symboles ne peuvent être additionnés ou soustraits. 


\subsection{Dégager le degré de concentration pour chaque section} échantillonnale - comprendre la circulation des idées selon les personnes au sein d'une région

L'opération précédente ne permet pas, non plus, d'identifier la relation entre les idées et les personnes pour chaque région. Un quatrième tableau a été développé afin de montrer les tendances générales des positions à l'égard d'une idée par journal. Ce tableau permet, par exemple, de savoir si les positions du Journal X ressemblent à celles du JournalY. Plus les tendances se ressemblent, plus il y aura déconcentration des idées, donc plus il sera possible de dire que les idées sont homogènes parmi les individus d'une région donnée. À l’inverse, plus les tendances se distinguent, plus il y aura concentration des idées, donc plus il sera possible de dire que les idées sont hétérogènes parmi les personnes de la région à l'étude.

Lorsqu'une idée qui est en relation avec les personnes est associée à un ton plutôt positif, c'est-à-dire que le pourcentage dans la colonne «Ton positif » est au moins de $61 \%$, elle sera représentée par une flèche qui se dirige vers la gauche $(-)$. Lorsqu'une idée prend un ton plutôt négatif, c'est-à-dire que le pourcentage dans la colonne "Ton négatif » est au moins de $61 \%$, elle sera représentée par une flèche qui se dirige vers la droite $(\rightarrow)$. Lorsqu'une idée se présente avec un ton ni positif, ni négatif, c'est-à-dire que le pourcentage dans l'une des deux colonnes se situe entre 40 et $60 \%$ et que le pourcentage dans l'autre colonne équivaut au pourcentage opposé, la flèche est bidirectionnelle $(\leftrightarrow)^{22}$.

\begin{tabular}{|c|c|c|}
\hline \multicolumn{3}{|c|}{$\begin{array}{c}\text { Tableau } 4 \\
\text { Prise de position à l'égard des thèmes selon le journal et l'année de parution }\end{array}$} \\
\hline Idées & Journal X de Ville X 1960 & Journal Y de Ville X 1960 \\
\hline IDÉE X & $\leftarrow$ & $\leftrightarrow$ \\
\hline IDÉE Y & $\rightarrow$ & $\leftarrow$ \\
\hline IDÉE Z & $\rightarrow$ & $\rightarrow$ \\
\hline \multicolumn{3}{|c|}{$\begin{array}{l}\text { Flèches fictives à des fins d'illustration seulement, mais en correspondance avec le tableau } 3 \text { : } \\
-=\text { tendance plutôt positive } ; \rightarrow=\text { tendance plutôt négative } ; \leftrightarrow=\text { tendance indiscernable }\end{array}$} \\
\hline
\end{tabular}

22 Les flèches dans le tableau 4 correspondent aux données du tableau 2. 
Dans les circonstances où un journal traite d'un sujet et l'autre ne l'aborde pas, le fait sera indiqué pour le premier journal et on trouvera une flèche bidirectionnelle pour le deuxième journal. Cette opération permettra de respecter l'importance de cette idée pour un groupe et elle n'imposera pas une position au deuxième groupe - une flèche bidirectionnelle marquera donc toujours l'impossibilité d'identifier une idée à un groupe-position.

\subsection{Comprendre les problèmes liés à notre méthode}

Les opérations que nous avons décrites jusqu'ici ne circonscrivent pas l'ensemble des informations qui circulent dans une société, c'est-à-dire qu'elles excluent de l'information. Par exemple, il est certain que les idées qui circulent en société, et qui sont en relation avec les personnes, ne peuvent généralement pas se prêter à une dichotomisation. Comme nous l'avons déjà expliqué, il est rare que, dans une société, une idée en relation avec les personnes se polarise à 50/50. Malgré le fait que nous croyons que notre méthode permet d'observer ce genre d'éventualité, nous reconnaissons que nous pourrions aussi lui reprocher de simplifier excessivement les informations repérées. Mais ces conséquences sont inhérentes aux devis qui sont construits pour la vérification d'hypothèses spécifiques. Le but n'est pas ici de tout dire sur ce qui se dit dans une société, mais de vérifier si les problématiques politiques évoluent vers l'homogénéisation. Notre opérationnalisation, comme celle de Laflamme (2000), vise une compréhension générale de la circulation des idées et des personnes, voire une compréhension macrologique de l'ensemble sociétal. Ainsi, les informations présentées dans les tableaux précédents ne doivent pas être analysées en tant qu'unités informationnelles, mais plutôt dans un tout intégré ${ }^{23}$.

Malgré toutes les critiques qu'on pourrait formuler, il est important de ne pas oublier que ce genre d'opération assure que les thèmes sont systématiquement vérifiés et évalués, ce qui protège contre la surreprésentation des thèmes, de même que contre les dérives qui pourraient être associées à une analyse strictement herméneutique ou encore, à une démarche intuitive.

23. Afin d'atténuer les effets de la simplification des informations liée à la méthodologie, nous avons mis en annexe dans le travail original, une description des idées qui occupent plus de $1 \%$ du nombre total d'idées qui se dégagent d'un journal en particulier (Roger Gervais, Les effets de la mondialisation..., op. cit). 


\section{Résultats de la collecte de données}

Notre corpus est constitué de 3610 articles, desquels nous avons dégagé 162 thèmes. Mille trois cents quarante et un (1 341) articles ont été recensés pour la région de Sudbury pour 1960 et 2000, soit 571 dans The Sudbury Star en 1960, 122 dans l'Ami du peuple en 1960, 518 dans The Sudbury Star en 2000 et 130 dans Le Voyageur en 2000. Comme le veut la méthodologie utilisée, les articles ont été répartis, dans un premier temps, selon leur catégorie géopolitique.

\begin{tabular}{|c|c|c|c|c|}
\hline \multicolumn{5}{|c|}{$\begin{array}{c}\text { Tableau } 5 \\
\text { Répartition des articles recensés selon la catégorie géopolitique pour } \\
\text { Sudbury, } 1960 \text { et } 2000\end{array}$} \\
\hline \multirow{3}{*}{$\begin{array}{l}\text { Catégories } \\
\text { géopolitiques }\end{array}$} & \multicolumn{4}{|c|}{ Pourcentage de fiches selon le journal } \\
\hline & \multicolumn{2}{|c|}{1960} & \multicolumn{2}{|c|}{2000} \\
\hline & The Sudbury Star & Ami du peuple & The Sudbury star & Le Voyageur \\
\hline International & $36 \%$ & $30 \%$ & $16 \%$ & $4 \%$ \\
\hline National & $25 \%$ & $40 \%$ & $27 \%$ & $8 \%$ \\
\hline Provincial & $19 \%$ & $15 \%$ & $24 \%$ & $13 \%$ \\
\hline Régional & $20 \%$ & $15 \%$ & $33 \%$ & $75 \%$ \\
\hline Total (N) & $100 \%(571)$ & $100 \%(122)$ & $100 \%(518)$ & $100 \%(130)$ \\
\hline
\end{tabular}

À la lecture du tableau 5, nous faisons deux constats en ce qui a trait au Sudbury Star: en 1960, $35 \%$ de tous les articles recensés dans ce journal, traitent de sujets internationaux ; par ailleurs, plus les articles traitent de sujets régionaux, moins le nombre d'articles est nombreux. L'Ami du peuple suit une tendance semblable : les questions nationales présentent plus grande part des articles, soit $40 \%$ du nombre total des articles recensés.

En 2000, cette tendance est inversée. Dans The Sudbury Star, les articles d'intérêt international sont les moins nombreux et les articles à vocation régionale sont les plus nombreux ; les thématiques nationales, toutefois, sont un peu plus communes, avec $27 \%$, que les thématiques provinciales avec $24 \%$; les sujets d'ordre provincial sont moins fréquents que ceux qui concernent la région, avec $33 \%$ des articles. Dans Le Voyageur, cette tendance contraire est plus prononcée : $3 \%$ sont d'intérêt international, $8 \%$ d'intérêt national, $13 \%$ provincial et, 
ensuite, avec un bond considérable, $75 \%$ d'intérêt régional. Ce dernier ensemble représente donc les trois quarts des articles recensés dans ce journal.

En ce qui concerne les idées repérées en 1960, pour The Sudbury Star, 1123 idées ont été examinées ; pour l'Ami du peuple, le nombre d'idées se chiffre à 267. En tout, 47 thèmes occupent plus de $1 \%$ de la totalité des idées qui se dégagent de chacun des deux journaux. Dans The Sudbury Starde 1960, ces 47 thèmes ont été présentés avec un ton positif à 495 reprises et avec un ton négatif à 391 reprises. Dans l'Ami du peuple, les 47 thèmes ont été présentés avec un ton positif à 122 occasions et 84 fois avec un ton négatif. Aucun thème ne représente plus de $10 \%$ du nombre total des articles recensés.

Huit cent vingt-six (826) idées ont été dégagées pour la région de Sudbury en 2000. De ce nombre, 613 idées ont été repérées à partir du Sudbury Staret 213, à partir de Le Voyageur. En tout, 34 thèmes occupent plus de $1 \%$ du total des idées de l'un des deux journaux. Dans ces 34 thèmes, 295 idées issues du Sudbury Star étaient présentées avec un ton positif, contre 276 avec un ton négatif. Un thème dans Le Voyageur et un thème dans The Sudbury Star en 2000 occupent entre 10 et $19 \%$ du total d'idées du journal. Pour Le Voyageur, 155 idées avaient un ton positif et 52 , un ton négatif.

\begin{tabular}{|c|c|c|c|c|}
\hline \multicolumn{5}{|c|}{$\begin{array}{c}\text { Tableau } 6 \\
\text { Répartition des articles recensés selon la catégorie géopolitique pour } \\
\text { Toulouse, } 1960 \text { et } 2000\end{array}$} \\
\hline \multirow{3}{*}{$\begin{array}{l}\text { Catégorie } \\
\text { géopolitique }\end{array}$} & \multicolumn{4}{|c|}{ Pourcentage de fiches selon le journal } \\
\hline & \multicolumn{2}{|c|}{1960} & \multicolumn{2}{|c|}{2000} \\
\hline & $\begin{array}{c}\text { La Dépêche du } \\
\text { Midi }\end{array}$ & $\begin{array}{c}\text { La Croix de } \\
\text { Toulouse }\end{array}$ & $\begin{array}{c}\text { La Dépếche du } \\
\text { Midi }\end{array}$ & $\begin{array}{c}\text { La Croix du } \\
\text { Midi }\end{array}$ \\
\hline $\begin{array}{l}\text { Internationale } \\
\text { Nationale } \\
\text { Provinciale } \\
\text { Régionale } \\
\text { Total (N) }\end{array}$ & $\begin{array}{c}58 \% \\
32 \% \\
\mathrm{~s} / \mathrm{o} \\
10 \% \\
100 \%(650)\end{array}$ & $\begin{array}{c}52 \% \\
18 \% \\
s / o \\
30 \% \\
100 \%(382)\end{array}$ & $\begin{array}{c}18 \% \\
38 \% \\
\text { s/o } \\
44 \% \\
100 \%(751)\end{array}$ & $\begin{array}{c}5 \% \\
10 \% \\
\text { s/o } \\
85 \% \\
100 \%(486)\end{array}$ \\
\hline $\begin{array}{l}\text { La c } \\
\text { Toul }\end{array}$ & . & gouvernen & $\begin{array}{l}\text { tinente pou } \\
\text { du pays. }\end{array}$ & n de \\
\hline
\end{tabular}


Dans la région de Toulouse, 2269 articles ont été recensés : 650 articles dans La Dépếche du Midi et 382 dans La Croix de Toulouse, en 1960; 751 dans La Dépéche du Midi et 486 dans La Croix du Midi, en 2000. Ces articles ont aussi été répartis selon leur catégorie géopolitique.

En examinant le tableau 6, nous constatons qu'en 1960,58\% de tous les articles recensés dans La Dépêche du Midi, traitent de sujets internationaux et que plus nous nous déplaçons vers les articles qui traitent de sujets régionaux, moins le nombre d'articles est élevé. $L a$ Croix de Toulouse, par contre, a $52 \%$ d'articles qui traitent de questions internationales, contre $18 \%$ qui concernent les questions nationales et $30 \%$ qui touchent à la région. Cette distribution fait en sorte que le régional occupe toujours moins de place que l'international, mais plus de place que le national. En 2000, la distribution est encore une fois renversée : les articles aux thèmes internationaux sont les moins nombreux (18\% pour La Dépéche du Midi et $5 \%$ pour La Croix du Midi), ceux à vocation nationale occupent une position intermédiaire (38\% pour La Dépếche du Midi et $10 \%$ pour La Croix du Midi) et les articles d'intérêt régional sont les plus nombreux (44\% pour La Dépêche du Midi et $85 \%$ pour La Croix de Toulouse). Ainsi, en suivant la variable indépendante, le pourcentage d'articles augmente au fur et à mesure qu'on s'approche de la catégorie géopolitique « régionale ».

Mille deux cent vingt-trois (1 223) idées ont été dégagées lors de la recension des fiches pour La Dépếche du Midi de 1960. Huit cent soixante-trois (863) d'entre-elles ont été identifiées dans La Croix de Toulouse. En tout, 39 thèmes occupent plus de $1 \%$ de la totalité des idées dans l'un des deux journaux. Dans La Dépếche du Midi, en 1960, ces 39 thèmes ont été présentés avec des idées au ton positif à 319 reprises et, avec des idées au ton négatif, à 498 reprises. Dans La Croix de Toulouse, les 39 thèmes ont été présentés avec des idées au ton positif à 386 occasions et avec des idées au ton négatif à 305 occasions. Un thème dans La Croix de Toulouse occupe, exceptionnellement, entre 10 et $19 \%$ du total des idées du journal.

En 2000, 1272 idées ont été dégagées de La Dépêche du Midi et 1016 de La Croix $d u$ Midi. En tout, 40 thèmes occupent plus de $1 \%$ de la totalité des idées qui circulent au sein d'au moins un de ces journaux. Parmi ces 40 thèmes, 516 idées ont un ton positif, 348, un ton négatif si nous examinons les fiches de La Dépếche du Midi. Pour La Croix du Midi, 707 idées ont un ton positif, 100, un ton négatif. Les idées qui 
composent un thème ne représentent jamais plus de $10 \%$ du total des idées repérées au sein d'un journal.

\section{Interprétation}

Le phénomène de la mondialisation suscite énormément de débats parmi les chercheurs en sciences sociales. Nous avons expliqué que, selon certains auteurs qui font état de comportements de masse ou encore de l'influence des médias, l'homogénéisation culturelle est théoriquement plausible. Selon d'autres, qui observent par exemple la formation de contre-cultures ou l'accentuation de l'individualisme, il serait préférable de parler de régionalisation, de différenciation ou même d'éclatement des cultures. D'aucuns avancent plutôt que ces deux pôles devraient être compris comme étant complémentaires. Quatre hypothèses ont découlé de cette polémique.

\subsection{Une société en voie d'homogénéisation?}

La première hypothèse veut que si le discours de l'homogénéisation est vrai, nous devrions observer plus de déconcentration en 2000, qu'en 1960, et ce, tant pour Toulouse que pour la Ville du Grand Sudbury.

\begin{tabular}{|c|c|c|c|c|c|c|c|c|c|}
\hline \multicolumn{10}{|c|}{$\begin{array}{c}\text { Tableau } 7 \\
\text { Fréquence des degrés de concentration selon les groupes associés aux } \\
\text { journaux sudburois }\end{array}$} \\
\hline \multicolumn{10}{|c|}{ Telolle al conoenuration } \\
\hline 5 & $10+$ & +1 & $+4+3$ & (1) & Pry & $+x+2$ & $4-2$ & $-2+$ & $\sqrt{2}$ \\
\hline $0-10$ & $10-20$ & $20-30$ & $30-40$ & 4050 & 5060 & $60-70$ & 7080 & 60.90 & $99-100$ \\
\hline \multicolumn{2}{|c|}{$\begin{array}{l}\text { Concentration / } \\
\text { Déconcentration }\end{array}$} & \multicolumn{2}{|c|}{$\begin{array}{c}\text { The Sudbury Star } \\
1960\end{array}$} & \multicolumn{2}{|c|}{$\begin{array}{c}\text { Ami du peuple } \\
1960\end{array}$} & \multicolumn{2}{|c|}{$\begin{array}{c}\text { The Sudbury Star } \\
2000\end{array}$} & \multicolumn{2}{|c|}{$\begin{array}{l}\text { Le Voyageur } \\
2000\end{array}$} \\
\hline \multicolumn{2}{|c|}{$\begin{array}{c}- \\
-+ \\
+- \\
+- \\
+\end{array}$} & \multicolumn{2}{|c|}{$\begin{array}{c}19 \\
4 \\
6 \\
7 \\
10\end{array}$} & \multicolumn{2}{|c|}{$\begin{array}{c}28 \\
7 \\
0 \\
3 \\
3\end{array}$} & \multicolumn{2}{|c|}{$\begin{array}{c}17 \\
2 \\
6 \\
6 \\
3\end{array}$} & \multicolumn{2}{|c|}{$\begin{array}{c}26 \\
0 \\
2 \\
1 \\
2\end{array}$} \\
\hline
\end{tabular}

Le tableau 7 montre que, pour la région sudburoise, les idées sont plus souvent très déconcentrées ( - ) au sein des groupes, quel que soit le 
journal ou l'année, et qu'il y a toujours une faible proportion de problématiques politiques concentrées $(+)$. Le degré d'homogénéisation est donc toujours très important. La proportion, toutefois, n'est ni variable, ni omniprésente, ce qui signifie que la thèse de l'homogénéisation ne résiste pas à la vérification, en ce qui a trait à la région de Sudbury.

\begin{tabular}{|c|c|c|}
\hline \multicolumn{3}{|c|}{ Tableau 8} \\
Fréquence des degrés de concentration dans la région de Sudbury \\
\hline Concentration / & Sudbury 1960 & Sudbury 2000 \\
Déconcentration & 31 & 22 \\
\hline- & 11 & 6 \\
+- & 5 & 6 \\
+ & & \\
\hline & & \\
\hline
\end{tabular}

Le tableau 8, qui présente les occurrences où les deux journaux partagent, au même moment, une position semblable $(-)$, différente $(+)$ ou indiscernable $(+-)$ à l'égard d'une idée, fait état d'une importante déconcentration des idées qui circulent dans la région de Sudbury en 1960 et en 2000. Cette tendance, qui est semblable à celle des journaux pris séparément (tableau 7), témoigne d'une homogénéisation, mais aussi d'une coprésence d'hétérogénéisation. Par conséquent, l'hypothèse de l'homogénéisation n'est pas vérifiée, ici non plus.

L'analyse des tableaux 9 et 10 oblige à tirer les mêmes conclusions pour la région de Toulouse. Cependant, une augmentation notable d'idées déconcentrées (corollaire d'une quasi-absence des idées non déconcentrées au sein de $L_{a}$ Croix du Midi de 2000, en comparaison à $L a$ Croix de Toulouse de 1960), invite à une certaine prudence : y a-t-il ici quelques preuves d'homogénéisation? Ces résultats sont possiblement liés au fait que le journal tend à informer davantage ses lecteurs d'activités et d'événements culturels que de débats politiques. Toutefois, nous ne croyons pas que cette prudence devrait être exagérée puisque le tableau 10 révèle que les positions à l'égard d'idées politiques suivent celles de La Dépêche du Midi de 2000 et que la tendance est stable.

Les tableaux 9 et 10 indiquent qu'il est impossible d'affirmer que la région toulousaine devient plus homogène. Vraisemblablement, l'évolution de la société ne transforme pas le degré de concentration ou 
de déconcentration des idées dans les régions de Sudbury et de Toulouse. Cela ne veut pas dire que les idées ne changent pas. Cela indique tout simplement que, même si les idées changent, il y a généralement consensus à leur égard parmi les personnes qui habitent ces deux régions. De plus, ce consensus n'impose pas une absence totale de différenciation; il y a, en effet, une certaine marginalisation, espace pour l'évolution, pour la transformation des idées.

\begin{tabular}{|c|c|c|c|c|c|c|c|c|}
\hline & $\begin{array}{l}\text { Fré } \\
\text { les g }\end{array}$ & $\begin{array}{l}\text { nce d } \\
\text { tpes a }\end{array}$ & $\begin{array}{l}\text { Tabl } \\
\text { s deg } \\
\text { socié }\end{array}$ & $\begin{array}{l}\text { u } \\
\text { de c } \\
\text { ux jo }\end{array}$ & $\begin{array}{l}\text { cent } \\
\text { laux }\end{array}$ & $\begin{array}{l}\text { on } \\
\text { dlousa }\end{array}$ & & \\
\hline (i) & U: & terh & tedeg & entrot & & & & (3) \\
\hline$-4 \mid t^{-4}$ & + & $t+1$ & 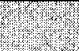 & Fit & $+4+2$ & +5 & $-x+$ & S-3 \\
\hline $10-20$ & $20-30$ & 3040 & 4050 & $50-60$ & 6070 & 7080 & 80,90 & $99-100$ \\
\hline $\begin{array}{l}\text { Concentration / } \\
\text { Déconcentration }\end{array}$ & $\begin{array}{l}\text { La Dép } \\
\text { Mid }\end{array}$ & $\begin{array}{l}\text { che du } \\
1960\end{array}$ & $\begin{array}{l}\text { LaC } \\
\text { Toulo }\end{array}$ & $\begin{array}{l}\text { ix de } \\
\text { e } 1960\end{array}$ & $\begin{array}{l}\text { La Déf } \\
\text { Mid }\end{array}$ & 2000 & $\begin{array}{l}\text { LaC } \\
\text { Toulor }\end{array}$ & $\begin{array}{l}\text { oix de } \\
\text { se } 2000\end{array}$ \\
\hline $\begin{array}{c}- \\
\ldots+ \\
+- \\
++- \\
+\end{array}$ & & & & & & & & $\begin{array}{l}2 \\
2 \\
0 \\
0 \\
1\end{array}$ \\
\hline
\end{tabular}

Tableau 10

Fréquence des degrés de concentration dans la région de Toulouse

\begin{tabular}{|c|c|c|}
\hline $\begin{array}{c}\text { Concentration / } \\
\text { Déconcentration }\end{array}$ & Toulouse 1960 & Toulouse 2000 \\
\hline- & 28 & 29 \\
+- & 7 & 9 \\
+ & 4 & 2 \\
\hline
\end{tabular}

La diminution des thématiques abordées pour la région de Sudbury de 1960 à 2000, soit de 49 à 34, pourrait indiquer une évolution vers l'homogénéisation - nous pourrions, en effet, concevoir que plus les individus pensent pareillement, moins il y aura de thèmes différents en circulation dans un groupe donné, alors plus il y aura de ressemblance. 
Toutefois, nous ne sommes pas convaincus que la différence soit suffisamment importante puisque le nombre d'idées qui circulent arrive toujours à composer plus de 30 thèmes.

\subsection{Une société en voie d'hétérogénéisation?}

La deuxième hypothèse que nous cherchions à vérifier est celle de la thèse de l'hétérogénéisation selon laquelle, si elle s'avère vraie, nous devrions observer plus de concentration en 2000 qu'en 1960, et pour la ville de Toulouse, et pour la Ville du Grand Sudbury. En nous reportant aux tableaux 7 à 10, nous constatons que cette tendance vers l'hétérogénéisation est aussi invisible. En effet, si nous considérons que la faible différence entre les idées qui circulent dans The Sudbury Star pour 1960 et celles qui circulent dans les autres journaux est négligeable, attribuable plutôt au hasard, le degré d'hétérogénéisation dans ces régions reste plus ou moins stable à travers le temps. Nous tirons cette conclusion du fait que, quoique les thèmes soient plus variés dans The Sudbury Star, les positions qui y sont prises ressemblent grandement à celles de L'Ami du peuple.

\subsection{Une mondialisation homogénéisante ou hétérogénéisante?}

La troisième hypothèse suggère que si la mondialisation fait en sorte que l'homogénéisation est mondialisation, nous devrions observer plus de similitudes en 2000 entre Toulouse et la Ville du Grand Sudbury, qu'en 1960. La quatrième annonce l'opposé. À ne se fier qu'aux résultats présentés dans les tableaux 5 et 6 , l'idée d'une homogénéisation mondiale est difficilement concevable puisque plus nous avançons dans le temps, moins les journaux consacrent d'articles aux événements internationaux. Les idées entre deux nations, plus précisément entre deux régions, deviennent homogènes grâce à quelle intervention, sinon par la recherche et par la diffusion des idées de l'Autre? Ces données donnent donc l'impression que plus la société évolue, moins il y a d'homogénéisation. Toutefois, elles ne permettent pas de faire de cette impression une véritable conclusion, et ce, pour deux raisons. Premièrement, cette différence pourrait être liée aux années que nous avons choisi d'étudier. En 1960, les préoccupations de nature internationale sont peut-être plus nombreuses puisque nous retrouverons, par exemple, une quantité importante d'articles qui 
traitent de problèmes internationaux comme la Guerre froide ou la situation défavorable des pays d'Afrique. En 2000, les événements internationaux paraissent moins mouvementés et moins préoccupants de par le fait qu'ils sont moins manifestes. Deuxièmement, cette différence pourrait être liée à l'arrivée des journaux qui ciblent beaucoup plus les nouvelles nationales et internationales, comme The Globe \& Mail à Sudbury, Le Monde à Toulouse, ou, encore, par l'influence d'Internet. Ne disposant pas des ressources de ces grandes institutions médiatiques, il devient plus rentable pour les périodiques régionaux de se concentrer sur le régional. Quelle qu'en soit la raison, nous ne pouvons pas, sur ce point, tirer de conclusion ferme à partir de nos données.

Nous pourrions aussi être porté à croire que l'analyse de la relation entre les idées et les personnes pour chaque journal régional et pour chaque région, puisqu'elle est d'une relation sans évolution, n'accrédite pas l'hypothèse d'une mondialisation homogénéisante. Pourtant, l'analyse de cette relation ne donne pas accès à ce genre d'observation puisque l'objectif du modèle est de favoriser une compréhension des idées et des personnes comme un tout intégré; si nous voulons observer les ressemblances et les différences parmi les idées des deux régions, ce sont les unités informationnelles (les idées), qui composent cette relation, ce tout, qui doivent être comparées.

La comparaison des idées qui circulent entre deux régions peut être inquiétante puisque nous n'avons retenu que les idées qui occupent au moins $1 \%$ des totaux - rappelons que ce seuil d'acceptation a été établi afin de distinguer les idées qui ont une pertinence tangible de celles qui sont plutôt contingentes. Mais si nous avons choisi d'effectuer un tel tri, c'est pour la simple raison que comparer les idées récurrentes avec celles qui sont accidentelles biaiserait les résultats en gonflant le degré d'homogénéisation : par exemple, évoquer les développements technologiques en aviation à Sudbury pourrait correspondre à un partage d'idée avec Toulouse qui le mentionne plus de 20 fois, alors qu'on sait bien que le rapport qu'entretient chacune de ces villes avec l'aviation n'est pas du tout semblable.

Il est, par ailleurs, délicat de comparer entre elles seulement les idées qui occupent $1 \%$ ou plus du total d'idées au sein d'un journal puisque cette procédure ne tient compte que des idées qui ont une certaine valeur numérique et ignore les autres ; ce qui résulte possiblement de facteur comme la brièveté de la période étudiée. Toutefois, ce genre de 
comparaison est plutôt sage par rapport au premier puisque le seuil est maintenu pour les deux régions.

Cela étant dit, le travail de comparaison permet de constater que, parmi les 63 thèmes, 40 ne sont présents que dans une des deux régions, tandis que 23 sont présents dans les deux régions, et cela, dans au moins un journal. Le degré de différence entre les deux régions est donc très élevé, mais le degré de ressemblance n'est pas négligeable.

La comparaison des thèmes en 2000 montre que 38 des 56 thèmes ne sont présents que dans une des deux régions, tandis que 18 de ces 56 thèmes sont présents dans au moins un journal dans chaque région. Le degré de différence est toujours plus important, mais le degré de ressemblance n'est pas négligeable.

La comparaison des idées entre les deux régions révèle donc que, quelle que soit l'année, il y a une plus grande proportion d'idées qui sont propres à une région que d'idées qui sont partagées. Par conséquent, et contrairement à ce que disent les données sur les régions, le degré d'hétérogénéisation est plus élevé que le degré d'homogénéisation, sans qu'il y ait négation de la seconde. Et cette dynamique reste stable dans le temps.

Pour ce qui est des hypothèses trois et quatre, les données de cette recherche indiquent qu'elles ne sont pas confirmées : il n'y a évolution ni vers l'homogénéisation, ni vers l'hétérogénéisation.

\subsection{Implication de ces résultats}

Cette analyse permet de faire quatre constats.

Premièrement, l'ensemble des résultats démontre que la relation entre les idées et les personnes, lorsqu'elle est comprise sur le plan régional, fait place à une homogénéité importante. Ce degré d'homogénéisation est logique pour des régions qui connaissent peu de révolutions, peu de chaos sociaux, donc une relative harmonie sociale. Il est donc possible de penser qu'une hétérogénéité ou une discordance trop élevée rendrait le rassemblement communautaire difficile et témoignerait d'une région chaotique $^{24}$. L'analyse de milieux comme Bagdad ou Kaboul pourrait servir à vérifier cette hypothèse. 
Deuxièmement, le fait que cette homogénéité n'est pas omniprésente pourrait expliquer, en partie $e^{25}$, l'évolution des idées au sein des régions entre les années étudiées. Une société qui connaît une assez grande harmonie sociale n'est pas nécessairement une société entièrement homogène ou qui n'est que pure reproduction. Des opinions différentes circulent dans ces régions et ces régions évoluent néanmoins.

Troisièmement, ces résultats montrent aussi que la dynamique homogénéité/hétérogénéité, lorsque comprise dans une comparaison de régions, et dans une optique internationale, est différente de la dynamique régionale, la relation entre les idées et les personnes faisant place à une plus grande hétérogénéité. Ce genre de dynamique montre que l'individualité régionale est toujours possible malgré le fait qu'au sein de ces régions, l'homogénéisation est importante. Pareillement, le particularisme régional ne signifie pas qu'il n'y ait pas de ressemblance entre les régions - les grands thèmes sociétaux sont en partie nécessaires à la structuration ${ }^{26}$ d'une région.

Quatrièmement, à la lumière de ces analyses, la dynamique homogénéité/hétérogénéité ne peut pas être dépendante de la mondialisation puisque celle-ci n'est pas indissociable de celle-là. Il n'est possible de dire ni que la "domination américaine » des médias de masse ou l'accroissement de l'utilisation des médias de masse a pour corollaire l'homogénéisation, ni que l'appropriation des médias de masse par d'autres cultures cause une différenciation généralisée. Ainsi, de la même manière que la mondialisation ne rend pas équivalents tous les médias de masse, ni l'un, ni l'autre de ces phénomènes ne renvoie à une dynamique homogénéisation/hétérogénéisation. Une appréhension plus complexe du phénomène est nécessaire afin de bien saisir les interactions entre des phénomènes d'une aussi grande envergure.

\section{Conclusion}

La mondialisation suscite encore des débats parmi les chercheurs en sciences sociales et notre recherche a voulu intervenir dans l'un d'eux,

25. Il est important de tenir compte du fait que la concentration n'équivaut pas à la différenciation. D’autres éléments peuvent contribuer à expliquer le phénomène de concentration.

26 Ce terme doit être compris à partir de sa définition littérale et non d'après son acception sociologique. 
celui qui s'interroge à savoir si la mondialisation est simultanément homogénéisation et différenciation. La thèse qui voudrait que les structures de pouvoir englobent l'ensemble de la société - donc rendent le tout homogène - semble toujours inapte pour comprendre le devenir des sociétés contemporaines, comme l'est celle qui voudrait que la culture des sociétés contemporaines véhicule des valeurs qui favorisent l'éclatement. Cette recherche s'est posée d'entrée de jeu entre ces deux thèses en s'alimentant à des logiques systémiques. Malgré le fait que, sur le plan théorique, les modèles de ce genre supposent qu'il importe d'analyser des dynamiques plurielles, ce sont surtout les aspects relationnels et dialectiques qui semblent être les plus utiles, tout simplement, parce qu'ils admettent l'existence de deux phénomènes contradictoires dans la coexistence d'entités paradoxales. Le modèle de la circulation des biens, des idées et des personnes nous a aussi permis d'aller un pas plus loin au plan pratique, en suggérant des degrés de concentration des problématiques politiques grâce auxquels il devenait possible de nous donner un objet analysable, les journaux.

En tenant compte des éléments régionaux (Paillart) et historiques (Miège), et en abordant de manière qualitative et quantitative des journaux régionaux, cette recherche montre qu'un modèle dialectique arrive mieux à saisir la dynamique entre des phénomènes, s'opposant à la fois à la thèse de l'homogénéisation et à celle de la différenciation. Elle montre que les relations causales ou les modélisations unilatérales ont peu de place dans ce genre d'analyse puisque la dynamique homogénéisation/différenciation n'est ni dépendante de la mondialisation, ni des médias de masse. Les concepts de dynamique et de dialectique seront donc plus adaptés à la compréhension de la réalité qu'est la mondialisation.

Il ne faut pas oublier, toutefois, que ces observations ne peuvent pas être présentées comme des conclusions définitives; cette recherche est exploratoire et elle appelle à des manipulations ultérieures. Par exemple, afin de mieux respecter la complexité du modèle et de la méthode, il conviendrait, dans une prochaine recherche, d'intégrer la dimension « biens ». L'analyse des problématiques politiques sur Internet, un média qui donne accès à divers genres d'information (l'information dite conforme à des normes de crédibilité, aux rumeurs, aux témoignages, aux opinions personnelles, etc.) pourrait permettre d'observer une dynamique homogénéité/hétérogénéité différente de celle des périodiques régionaux. Ou encore, l'analyse des nouvelles télévisées pourrait permettre de découvrir d'autres agencements de cette dynamique et, combinée à celle 
des journaux, pourrait assurer une meilleure représentativité de la problématique politique. La variable «temps » pourrait aussi affecter les résultats de cette présente recherche. Par exemple, l'ajout de périodes qui précèdent 1960 montrerait peut-être que la télévision ou la radio sont de plus grands homogénéisateurs que ne l'est Internet. Après tout, la télévision a été mal perçue à cet égard bien avant l'avènement d'Internet. De plus, malgré notre croyance qu'un écart de trois mois est suffisant pour observer le degré d'homogénéisation dans une région, il serait important d'étendre cette période pour la comparaison de deux régions au plan international en sachant que plus la période d'analyse est longue, moins sont déterminantes les idées les plus contingentes.

Étant donné que De la circulation des biens, des idées et des personnes au Canada (1981-1995) nous confronte à un degré d'homogénéisation qui, pour la nation, est semblable à celui que nous observons dans les régions, il serait peut-être important de vérifier si le modèle lui-même, de par sa nature, n'impose pas ce genre de résultats. Nous proposons donc d'appliquer le modèle à des régions où les structures sociales sont plus totalitaires, moins démocratiques ou à celles qui ne connaissent pas la concurrence entre les médias. Ces régions devraient témoigner d'une homogénéisation encore plus marquée. Observer autre chose que ce que le modèle a permis d'observer pourrait être indicatif des lacunes inhérentes au modèle lui-même.

On nous a aussi signalé que notre échelle de concentration ne permet pas l'analyse d'une hétérogénéité (de concentration) puisque le degré le plus concentré de notre échelle est de 50/50 - les idées étant concentrées au sein de deux groupes distincts témoignent plutôt de deux parts importantes d'homogénéisation et non pas de deux parts importantes d'hétérogénéisation. Cela fait en sorte que la possibilité d'idées individuelles ou d'idées contradictoires n'est pas observable par le recours à notre seule échelle. L'échelle de concentration mesure ainsi plutôt le degré de concentration des idées déconcentrées, récurrentes, que les idées en général. Afin de corriger cette faille, nous pourrions, par exemple développer davantage les informations présentées par les idées récurrentes à moins de $1 \%$ du total. Cela permettrait de mieux comprendre cette zone de hasard, à caractère arbitraire, voire hétérogène. Nous soulignons, par contre, que le fait même qu'il puisse y avoir une part importante d'idées récurrentes (preuve d'homogénéité) parmi cette hétérogénéité, quelle que soit la région ou l'époque étudiée, ne fait que renforcer nos prémisses théoriques d'une dynamique 
homogénéisation/hétérogénéisation. Par ricochet, cette dynamique continue à invalider les thèses alarmistes de l'homogénéisation ou de la différenciation.

Ces quelques pistes d'études futures mettent en évidence l'importance de continuer les recherches dans ce domaine. En raison de sa nature et de sa forme exploratoire, cette recherche est certes limitée et elle commande une interprétation prudente. Néanmoins, nous croyons avoir élaboré un travail qui pourrait servir de tremplin à des recherches subséquentes.

\section{Références}

Adorno, ThÉodor et Max Horkheimer, La dialectique de la raison : fragments philosophiques, Paris, Gallimard, 1974.

Baudrillard, Jean, Amérique, Paris, Grasset et Fasquelle, 1986.

Baudrillard, Jean, La transparence du Mal : Essai sur les phénomènes extrêmes, Paris, Galilée, 1990.

Berthoud, Gérald, "L'horizon d'une surveillance omniprésente ? ", Revue européenne des sciences sociales, Tome XL, $\mathrm{n}^{\circ} 123$, 2002, p. 155-168.

Boisvert, Yves, L'analyse postmoderniste : une nouvelle grille d'analyse socio-politique, Montréal, L'Harmattan Inc, 1997.

Boisvert, Yves, «Sortir du nihilisme : Quand la dictature du moi sert de bouée », dans Yves Boisvert et Lawrence Olivier (dir.), A chacun sa quête : essais sur les nouveaux visages de la transcendance, Québec, Presses de l'Université du Québec, 2000, p. 1-10.

Breton, Philippe, «La société de l'information : de l'utopie au désenchantement », Revue européenne des sciences sociales, Tome XL, n" 123, 2002, p. 35-39.

Castells, Manuel, La société en réseaux : l'ère de l'information, tome 1, traduit de l'anglais par Philippe Delamare, Paris, Fayard, 1998.

Gagnon, Bernard, «Le soi et le différent à l'âge de l'indifférence : la problématique de l'éducation ", dans Yves Boisvert et Lawrence Olivier (dir), À chacun sa quête : essais sur les nouveaux visages de la transcendance, Québec, Presses de l'Université de Québec, 2000, p. 86-103.

Gervais, Roger, Les effets de la mondialisation sur la relation entre les idées et les personnes : une comparaison entre les régions toulousaine et sudburoise, mémoire de maitrise, Sudbury, Université Laurentienne, 2005.

Guillén, Mauro, F., «Is Globalization Civilising, Destructive Or Feeble? A Critique of Five Key Debates In The Social Science Literature », Annual Review of Sociology, vol. 27, août 2002, p. 235-260.

Karnoouh, Claude, Adieu à la différence : essais sur la modernité tardive, Paris, Arcantère Karnoouh, 1993.

Lafay, Gérard, Michael Freudenberg, Colette Herzog et Deniz Ünal-Kesenci, Nations et mondialisation, Paris, ECONOMICA, 1999. 
100 NPSS, VOLUME 2, NUMÉR0 1, 2006

Laflamme, Simon, La societé intégrée : de la circulation des biens, des idées et des personnes, New York, Peter Lang, 1992.

Laflamme, Simon, De la circulation des biens, des idées et des personnes au Canada (1981-1995): un modèle macrologique relationnel, Paris/Sudbury, L'Harmattan/Prise de parole, 2000.

Laflamme, Simon et Ali Reguigui, Homogéneíté et distinction, Sudbury, Prise de parole, 2004. Luhmann, Niklas, The Reality of the Mass Media, California, Standford University Press, 2000.

Maingueneau, Dominique, Genèses du discours, Liège, Mardaga, 1984.

Miège, Bernard, "La société de l'information : toujours aussi inconcevable ", Revue européenne des sciences sociales, 'Tome XL, $\mathrm{n}^{\circ} 123,2002$, p. 41-54.

Morin, Edgar, Intraduction à la pensée complexe, Paris, ESF éditeur, 1990.

Paillart, Isabelle, "La société de l'information : une société de contradictions?", Revue européenne des sciences sociales, Tome XL, $\mathrm{n}^{\circ} 123,2002$, p. 55-63.

Teeple, Gary, Globalization and the Decline of the Social Reform: Into the Twenty-first Century, Aurora, Garamond Press, 2000.

Touraine, Alain, Pourrons-nous viure ensemble ? Égaux et différents, Paris, Librairie Arthème Fayard, 1997. 